\title{
Reversing disease
}

'It was recognised early in the course of the global epidemic of type 2 diabetes that variation in the prevalence of the disease among populations could be explained largely by relative weight gain. ${ }^{[1]}$ This quote is from a 2013 article that reported the results of an evaluation of associations between population-wide loss and gain in weight with diabetes prevalence, incidence and mortality, as well as cardiovascular and cancer trends, in Cuba over a 30-year interval. The global epidemic of type 2 diabetes is generally thought to be a result of the increasing incidence of obesity among all populations. If weight gain causes type 2 diabetes in susceptible individuals, would weight loss reverse the disease? A recent analysis in the $B M J^{[2]}$ would suggest that it can.

Louise McCombie and her colleagues ${ }^{[2]}$ from the University of Glasgow, Scotland, point out that type 2 diabetes is generally regarded as progressive and incurable. In the UK alone this disease now affects $5-10 \%$ of the population. The estimated national prevalence of type 2 diabetes in South Africa in 2012 was $9.5 \%,{ }^{[3]}$ so we are dealing with a similar problem. The current approach to treating this disease relies on frequent measurements of glycated haemoglobin (HbAlc) along with early introduction of drugs and some lifestyle recommendations, such as weight loss and exercise. While this approach has improved clinical outcomes to some extent, most patients still go on to develop vascular complications and their life expectancy is up to 6 years shorter than that of individuals without diabetes.

However, overseas at least (and I know among some pioneering practitioners locally) diabetes management is beginning to focus on reversing the underlying disease mechanism rather than treating symptoms and the long- and short-term consequences of the pathology. Current clinical guidelines focus on lowering blood glucose and $\mathrm{HbAlc}$, generally using antidiabetic drugs. There are 488 drugs (excluding insulins) currently licensed worldwide to treat type 2 diabetes, with 70 generic compounds. All these lower $\mathrm{HbA1c}$ and blood glucose significantly - but diabetics continue to suffer the consequences of the disease, become sick and die prematurely. To date, there have been no trials looking at the combination of drugs given along with optimal diet and lifestyle advice for weight control.

On the other hand, consistent evidence shows that weight loss is associated with extended life expectancy for people with diabetes and that weight loss of around $15 \mathrm{~kg}$ often produces total biochemical remission of type 2 diabetes, restoring beta-cell function. ${ }^{[4]}$ We now know that one of the main reasons for the pathology is the accumulation of ectopic fat in the liver and pancreas that impairs organ function, and that reversing this accumulation reverses the disease. McCombie et al. ${ }^{[2]}$ suggest that a simple approach to classifying remission as two normal tests (either blood glucose or $\mathrm{HbA1c}$ ) at least 2 months apart and regular annual follow-up should be sufficient to diagnose 'remission'.

However, although we are now starting to accept the idea that a diagnosis of diabetes need not be for life, few patients are achieving the weight loss required for remission. In Scotland, for example, results that included every patient in Scotland with diabetes showed that $<0.1 \%$ of those with type 2 diabetes were coded as being in remission in March 2017. McCombie et al. ${ }^{[2]}$ suggest that the main reason for this is that neither patients nor their doctors are focusing on trying to achieve remission. Indeed, they may be unaware that this can be achieved. There is wide doubt that people can sustain weight loss, despite evidence of structured approaches that have recorded rapid initial weight loss and then maintenance of weight loss of $12-15 \mathrm{~kg}$ for $>12$ months. ${ }^{[5]}$ For many, realising that losing weight will lose them the diagnosis of diabetes will be enough motivation to find a lifestyle and diet that allows them to do this. All doctors need to start looking at type 2 diabetes as a potentially reversible disease, so that their patients can be encouraged to do the same.

\section{Bridget Farham \\ Editor \\ ugqirha@iafrica.com}

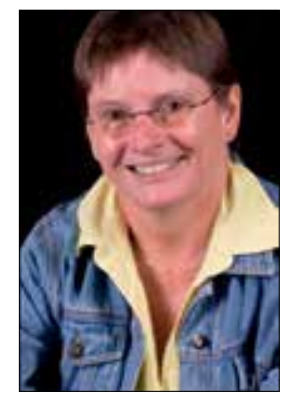

1. Franco M, Bilal U, Orduñez P, et al. Population-wide weight loss and regain in relation to diabetes burden and cardiovascular mortality in Cuba 1980 - 2010: Repeated cross sectional surveys and ecological comparison of secular trends. BMJ 2013:346:f1515. https://doi.org/10.1136/bmj.f1515

McCombie L, Leslie W, Taylor R, Kennon B, Sattar N, Lean MEJ. Beating type 2 diabetes into remission BMJ 2017:358:j4030. https://doi.org/10.1136/bmj.j4030

3. SEMDSA Type 2 Diabetes Expert Committee. SEMDSA 2017 guidelines for the management of type 2 diabetes mellitus. J Endocr Metab Diabetes S Afr 2017;22(1, Suppl 1):S1-S196. https://www.semdsa.

4. Lim EL, Hollingsworth KG, Aribisala BS, Chen MJ, Mathers JC, Taylor R. Reversal of type 2 diabetes Lim EL, Hollingsworth KG, Aribisala BS, Chen MJ, Mathers JC, Taylor R. Reversal of type 2 diabetes:
Normalisation of beta cell function in association with decreased pancreas and liver triacylglycerol. Diabetologia 2011;54(10):2506-2514. https://doi.org/10.1007/s00125-011-2204-7

5. Stevens S, Hollingsworth KG, Al-Mrabeh A, et al. Very low-calorie diet and 6 months of weight stability in type 2 diabetes: Pathophysiological changes in responders and nonresponders. Diabetes Care 2016;39(5):808-815. https://doi.org/10.2337/dc15-1942

S Afr Med J 2017;107(10):807. DOI:10.7196/SAMJ.2017.v107i10.12877 